\title{
Use of HIV Case Surveillance System to Design and Evaluate Site- Randomized Interventions in an HIV Prevention Study: HPTN 065
}

\author{
Deborah J. Donnell ${ }^{*}, 1$, H. Irene Hall ${ }^{2}$, Theresa Gamble ${ }^{3}$, Geetha Beauchamp ${ }^{1}$, Angelique B. Griffin ${ }^{4}$, \\ Lucia V. Torian ${ }^{5}$, Bernard Branson ${ }^{2}$ and Wafaa M. El-Sadr ${ }^{6}$, for the HPTN 065 (TLC-Plus) Study Team \\ ${ }^{I}$ Vaccine and Infectious Disease Division, Fred Hutchinson Cancer Research Center, Seattle, WA, USA \\ ${ }^{2}$ Division of HIV/AIDS Prevention, Centers for Disease Control and Prevention, Atlanta, GA, USA \\ ${ }^{3}$ FHI 360, Durham, NC, USA \\ ${ }^{4}$ Strategic Information Bureau, HAHSTA, District of Columbia Department of Health. Washington, D.C., USA \\ ${ }^{5}$ HIV Epidemiology and Field Services Program, NYC Department of Health, New York, NY, USA \\ ${ }^{6}$ ICAP-Columbia University and Harlem Hospital New York, NY, USA
}

\begin{abstract}
Introduction: Modeling studies suggest intensified HIV testing, linkage-to-care and antiretroviral treatment to achieve viral suppression may reduce HIV transmission and lead to control of the epidemic. To study implementation of strategy, population-level data are needed to monitor outcomes of these interventions. US HIV surveillance systems are a potential source of these data.

Methods: HPTN065 (TLC-Plus) Study is evaluating the feasibility of a test, linkage-to-care, and treat strategy for HIV prevention in two intervention communities - the Bronx, NY, and Washington, DC. Routinely collected laboratory data on diagnosed HIV cases in the national HIV surveillance system were used to select and randomize sites, and will be used to assess trial outcomes.

Results: To inform study randomization, baseline data on site-aggregated study outcomes was provided from HIV surveillance data by New York City and Washington D.C. Departments of Health. The median site rate of linkage-to-care for newly diagnosed cases was 69\% (IQR 50\%-86\%) in the Bronx and 54\% (IQR 33\%-71\%) in Washington, D.C. In participating HIV care sites, the median site percent of patients with viral suppression $(<400$ copies $/ \mathrm{mL}$ ) was $57 \%$ (IQR $53 \%-61 \%$ ) in the Bronx and 64\% (IQR 55\%-72\%) in Washington, D.C.

Conclusions: In a novel use of site-aggregated surveillance data, baseline data was used to design and evaluate site randomized studies for both HIV test and HIV care sites. Surveillance data have the potential to inform and monitor sitelevel health outcomes in HIV-infected patients.
\end{abstract}

Keywords: HIV, linkage-to-care, site randomized, surveillance, test and treat, viral load suppression.

\section{INTRODUCTION}

Evidence is accumulating that antiretroviral treatment of HIV-infected persons results in prevention of HIV transmission - that "treatment is prevention." Observational and ecological evidence suggest that antiretroviral treatment (ART) reduces the risk of transmission to HIV-uninfected persons $[1,2]$. Recent results from a randomized study (HPTN 052) established that ART initiation at CD4 counts between 350 and 550 cells $/ \mathrm{mm}^{3}$ in the HIV infected partner reduced HIV transmission by $96 \%$ to the uninfected partner in discordant couples [3]. Given this demonstration of reduced HIV transmission through use of ART, the "testand-treat" strategy to decrease spread of HIV in the community is receiving serious operational consideration.

*Address correspondence to this author at the Fred Hutchinson Cancer Research Center, 1100 Fairview Ave N, M2-C200, PO Box 19024, Seattle WA 98109, USA; Tel: (206) 667-5661; Fax: (206) 667-4378;

E-mail: Deborah@fhcrc.org
This strategy combines expanded HIV testing to identify undiagnosed HIV infection with prompt linkage-to-care and initiation of ART to lower HIV viral load (VL) levels [4-8]. People aware of their HIV infection have been shown in several studies to adopt safer behaviors [9], and HIVinfected individuals adhering to effective treatment achieve VL suppression in the blood stream and genital tract and become less infectious, resulting in decreased HIV transmission $[3,10,11]$. In the United States, an estimated $21 \%$ of people living with HIV do not know they are infected [12], less than 50\% of Americans aged 18-64 report having been tested for HIV [13], and linkage-to-care and retention in care are suboptimal [14-18]. Nonetheless the test-and-treat strategy is compelling because it has the potential to lower infection risk for the entire population. Projects to assess the feasibility of achieving high coverage of testing, treatment, and viral suppression are critical for understanding how best to improve health outcomes among those with HIV infection and decrease their risk of transmission. 
Methods and designs for efficiently measuring the effectiveness of test and treat programs at the community level are urgently needed. Randomized clinical trials remain the gold standard for proving efficacy of new prevention interventions in a research setting with high fidelity of implementation and evaluation. As proven prevention interventions move from randomized clinical trials into implementation, the primary question changes from establishing efficacy in a clinical trial setting to evaluating effectiveness in program settings and, ultimately, in populations.

U.S. HIV surveillance systems are increasingly able to reliably monitor indicators of access to care for identified cases of persons living with HIV/AIDS, as laws mandating name based reporting of newly detected cases are implemented, along with mandatory reporting of HIV viral load and CD4 counts from laboratories within the jurisdiction to the surveillance systems $[19,20]$. The surveillance systems thus have the potential to be used as a platform for evaluating interventions in persons known to be HIV-infected, building on current surveillance evaluations of linkage to care and retention in care $[17,21]$.

The HPTN 065 (TLC-Plus) study was designed to determine the feasibility of implementing an "Enhanced Test, Link to Care, Plus Treat" approach for HIV prevention in the United States. The study incorporates two siterandomized components to evaluate the effectiveness of financial incentives (FIs) for increasing linkage-to-care and VL suppression in two communities: the Bronx, NY and Washington, D.C. The study proposes a novel use of surveillance data to assess aggregate site outcomes in HIV infected patients accessing testing and care. Surveillance data was used to inform design and implementation of the site-randomized study components and will be used to assess the outcomes of the site level interventions. To our knowledge, HPTN 065 (TLC-Plus) is the first study to use HIV surveillance data to design and determine key HIVrelated health outcomes of interventions in a site-randomized trial.

In this paper we describe the use of baseline site aggregate data from surveillance systems in the Bronx, NY, and Washington, D.C. to design the financial incentive components of the study and conduct the randomization of the participating sites in the two study cities. Assessing baseline site aggregate measures provides a proof of concept for the future use of aggregate site data from surveillance systems for measuring the interventions' effectiveness in HPTN065.

\section{METHODS}

\section{HPTN 065 Study Design}

HPTN 065 (TLC Plus) is a feasibility study of an enhanced, link to care, plus treat approach to HIV prevention that incorporates five components addressing different operational aspects of implementing a test and treat strategy for HIV prevention: expanded HIV testing, use of FIs for linkage to care, use of FIs for viral load suppression, a behavioral intervention for HIV-infected patients, and a survey of patient and provider attitudes to ART treatment for prevention. The study initiated in 2010 in the Bronx, NY, and Washington D.C., and is designed to gather data to inform the implementation of strategies for increasing HIV testing and improving access and adherence to HIV treatment for HIV infected patients. Increased HIV testing is being implemented in inpatient and emergency room settings in participating hospitals. Financial incentives are being evaluated for enhancing both linkage to care and viral suppression for HIV-infected persons: these components will be assessed in HIV testing and HIV care sites in the Bronx, NY and Washington DC, using New York City and Washington D.C. surveillance data. In addition, Department of Health (DOH) HIV testing and surveillance data from four non-intervention cities (Houston, TX; Miami, FL; Chicago, IL; Philadelphia, PA) will be used in an ecological comparison. This paper describes the use of surveillance data to implement the two financial incentive components of the study.

\section{HIV Surveillance System}

Since 1982, all 50 U.S. states and the District of Columbia have reported AIDS cases to the Centers for Disease Control and Prevention (CDC) in a uniform format. In 1994, the CDC integrated national HIV case surveillance with AIDS case surveillance, at which time 25 states with confidential, name-based HIV surveillance started submitting HIV case reports to CDC. Over time, additional states adopted name-based HIV surveillance; all states had done so by April 2008. Cases are reported to the national HIV surveillance system at the CDC without identifying information. Assessments of potential duplicate case reports occur both at the state and national level; resolution of these duplicate reports occurs at the state level.

Data collected for HIV surveillance include demographic characteristics of persons diagnosed with HIV, as well as clinical, laboratory, and vital status information. Historically, information was collected only at the time of diagnosis of HIV, AIDS, and at the time of death. Over time, state and local laws and regulations required laboratories to report HIV-related diagnostic test information, including positive Western blot (WB) results, plasma VL test results and CD4 counts to local DOH. With laboratory reporting of all HIVrelated test results, it is now possible to determine the stage of disease at diagnosis (through CD4 cell count), to assess utilization of HIV care sites based on laboratory tests performed at the sites (identified by the provider that ordered the test), and to monitor CD4 counts and VL levels in their population of patients over time.

Laboratory reporting can also provide information regarding the patients' current address of residence and the location where they receive HIV care. Site information in the surveillance system allows aggregation of data by specific care site, which enables comparisons of the number of HIVinfected patients in care at individual sites, the CD4 counts and VL levels of their patient populations, and any changes related to interventions at specific sites.

Using surveillance systems to capture aggregate site outcomes requires high-quality surveillance data with complete capture of HIV-related laboratory results. The study is being conducted in Bronx, NY, and Washington, D.C., using surveillance data from the New York City and Washington D.C. DOH jurisdictions. New York City has had 
mandatory electronic submission of positive $\mathrm{WB}$ and all VL and $\mathrm{CD} 4$ values from all testing laboratories to the $\mathrm{DOH}$ since 2005. Completeness and accuracy of dates, values and demographic data of CD4 counts and VLs are validated in the field by chart review, by monitoring of laboratory volume and domain values for each test type, as well as the patient identifiers that are used for matching, and by periodic laboratory audits that compare in-house laboratory data with data received by the New York State Electronic Clinical Laboratory Reporting System (ECLRS). The laboratory quality control duties are shared between New York City and New York State. All laboratories that currently perform HIV-related testing are enrolled in ECLRS, because all laboratories that perform any testing within New York State or for New York State providers are required to be certified for each test by the NYS Clinical Laboratory Evaluation Program (CLEP). Each time that CLEP approves a new laboratory or a new test for an enrolled laboratory, the NYS DOH contacts the laboratory, establishes or modifies any reporting system as required, and closely monitors startup through a thorough certification process that involves both test and production transmissions. Once the laboratory has been certified, it is routinely monitored as described above.

The Washington, D.C. DOH has rapidly achieved almost complete electronic reporting of HIV-related laboratory results following a major investment in surveillance data systems beginning in 2008. Paper-based laboratory data is manually entered. Completeness and accuracy of dates, values and demographic data of CD4s and VLs are validated in the field by chart review, and systems for routine monitoring of laboratory reporting are being defined.

Strict federal and state confidentiality constraints limit the disclosure of data from name-based HIV/AIDS surveillance systems. Only de-identified aggregate data can be released from the system. Analysis and publication of surveillance data broken down by demographic categories, risk factors, and diagnostic or care provider are routine. Because HPTN 065 requires only site-aggregated data, it is an appropriate extension of routine practice to use site aggregate data for planning and evaluation of the financial incentive components of HPTN 065.

\section{Financial Incentive Intervention}

Two HIV-related interventions are being evaluated in two separate site-randomized components of HPTN 065: FI for increasing linkage-to-care for newly diagnosed cases and FI for increasing VL suppression for patients on ART. The duration of the interventions is two years at each site. Outcomes captured in surveillance data, aggregated within sites randomized to the two arms, will be used to assess the effectiveness of FIs.

\section{Site Selection}

For the FI components of HPTN 065, the goal was to recruit 20 test and 20 care sites per municipality. For HIV test site selection, HIV test sites in the Bronx and Washington D.C. with the highest number of newly diagnosed HIV infections in the previous calendar year were identified using the most recent surveillance year available at the time of site recruitment and selection. For HIV care site selection, care sites in the Bronx and Washington, D.C. with the highest number of HIV infected patients were identified from the surveillance data. If more than 20 sites were recruited, sites with higher volume were selected. Selection and recruitment of sites relied on close coordination with the DOH in New York City and Washington, D.C. and extensive cooperation and communication with testing and health provider organizations. Many of the largest providers of HIV health care provide both HIV testing and care, and were eligible as both test and care sites.

\section{Study Outcomes}

During study design, 2007 surveillance data from New York City and 2008 data from Washington D.C. were used to inform design parameters: i.e., to estimate size of site populations, study outcomes at baseline and intraclass correlation of outcomes across sites. At the time of study randomization, which occurred between October 2010 and March 2011, updated data - 2008 data from New York City and 2009 data from Washington D.C. - were used as baseline measures in conducting a restricted randomization of sites.

Linkage-to-Care measures: The study outcome for assessing linkage-to-care in HPTN 065 defines a case as linked to care when a VL or CD4 test result occurs within 3 months of an HIV-positive test at a participating test site. Siteaggregated surveillance data for 1) the number of newly diagnosed HIV-infected cases identified at a site and 2) the proportion of these cases linked to care within three months was used as baseline data for conducting the randomization of selected test sites.

Viral suppression measures: The study outcome for the VL suppression component of HPTN 065 defines a case with suppressed VL when VL is $<400$ copies/mL among HIVinfected patients in care (previous VL or CD4 count) at a participating site. The surveillance systems do not capture information about ART, thus the study outcome is assessed in all HIV-infected patients in care, irrespective of ART. The site-aggregated surveillance data for: 1) the number of HIVinfected patients with a care visit at the site in the corresponding calendar year, and 2) the proportion of those patients with VL suppression at the last visit were used as baseline data for randomization of the selected care sites.

\section{Randomization Procedures}

The goal of the restricted randomization procedure was to ensure balance between arms of two site characteristics that were likely highly predictive of study outcomes: 1) baseline case volume and 2) study outcomes at baseline [22]. Sites were randomized after they had obtained IRB approvals, and randomizations were conducted in blocks as IRB approvals were obtained. Each city and each component was randomized independently. To select a site randomization for a component and group of sites, all possible 1: 1 randomizations were computed and ranked for balance in the respective baseline number of cases and outcome (baseline linkage-to-care and viral load suppression, respectively) in each arm. Balance was computed using the following index for a given randomization into two groups of equal size, $R_{1}, R_{2}$ :

$I\left(R_{1}, R_{2}\right)=\left(V\left(R_{1}, R_{2}\right)\right)^{2}+\left(P\left(R_{1}, R_{2}\right)\right)^{2}$ 
where the site patient volumes, $\mathrm{V}$, and outcome proportions, $\mathrm{P}$, are divided into the two groups and $V\left(R_{1}, R_{2}\right)$ and $P\left(R_{1}, R_{2}\right)$ are the unequal variance t-statistics comparing mean volume and mean outcome proportion, respectively, between the two groups i.e.:

$$
V\left(R_{1}, R_{2}\right)=\frac{\mathrm{V}_{\mathrm{R}_{1}}-\mathrm{V}_{\mathrm{R}_{2}}}{\sqrt{\mathrm{s}\left(\mathrm{V}_{\mathrm{R}_{1}}\right)^{2}+\mathrm{s}\left(\mathrm{V}_{\mathrm{R}_{2}}\right)^{2}}} ; \quad P\left(R_{1}, R_{2}\right)=\frac{\mathrm{P}_{\mathrm{R}_{1}}-\mathrm{P}_{\mathrm{R}_{2}}}{\sqrt{\mathrm{s}\left(\mathrm{P}_{\mathrm{R}_{1}}\right)^{2}+\mathrm{S}\left(\mathrm{P}_{\mathrm{R}_{2}}\right)^{2}}}
$$

When randomizations were conducted in blocks, later blocks calculated the index including the previously randomized sites to ensure overall balance was maintained. In each block, the chosen randomization was selected at random from the $\mathrm{N}$ randomizations with highest rank $(\mathrm{N}$ varied with the number of sites being randomized). Where the number of sites was unequal, the number of sites in each arm could only differ by 1 .

Randomization for the linkage-to-care and the viral suppression components could be conducted independently, even though some sites operated as both test and care sites, as the two interventions occur at different times for an HIV infected patient: the linkage-to-care intervention is triggered by an HIV-positive test (likely before a person is in care), whereas the intervention for VL suppression is initiated amongst patients on antiretroviral therapy.

\section{RESULTS}

\section{Site Selection and Randomization}

In the Bronx, $27 \mathrm{HIV}$ test sites were identified and approached, of which 18 were ultimately recruited for participation in the study. In Washington D.C., 31 test sites were identified and 28 sites approached, of which 19 were recruited for the study (Fig. 1). Only 6 sites in the Bronx and 3 in Washington D.C. did not respond or declined participation. Test site randomization occurred from Bronx sites in February, 2011, using data from 2008 and for Washington D.C. in February and March 2011, using data from 2009.

Thirty-six care sites were approached in the Bronx, and 20 highest volume sites were selected for participation in the study. In Washington, D.C., 32 care sites were identified and 27 approached, of which 19 were recruited. Seven sites in the Bronx and 4 in Washington, D.C. did not respond or declined participation (Fig. 2). Care site randomization occurred in the Bronx in January 2011, and for Washington, D.C., in October 2010 and January 2011.

For either intervention, a site could only be randomized if they were able to implement the FI intervention within the patients attending that clinic and site data could be clearly identified within the surveillance system. Three test sites originally planned to be randomized as separate sites ultimately had to be combined because individual site data could not be reliably identified in surveillance. Five care sites also were combined either because of operational dependence (e.g. patients frequently scheduled at different clinics in the same provider system) or because centralized lab processing in the provider system meant data could not be clearly identified in surveillance systems. 12 of the selected sites in the Bronx and 11 in Washington D.C. are both test and care sites.

\section{Baseline Site Aggregate Data for Linkage-to-Care}

Surveillance data on the number of new diagnoses and linkage-to-care proportions used for selecting a randomization of the participating test sites is given in Table 1. Four sites in the Bronx and Washington, D.C. identified more than 50 newly diagnosed HIV cases in 2008 and 2009, respectively. An additional 5 sites in the Bronx and 9 sites in Washington D.C. identified between 10 and 50 newly identified cases, and the remaining 15 each found fewer than

\section{Bronx NY}

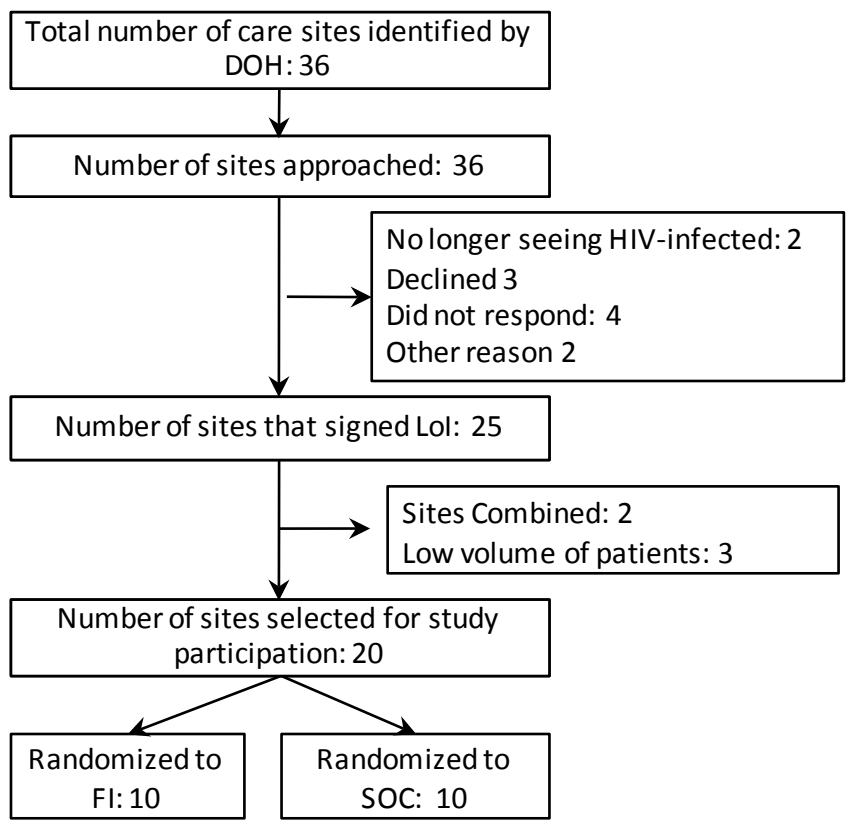

Washington D.C.

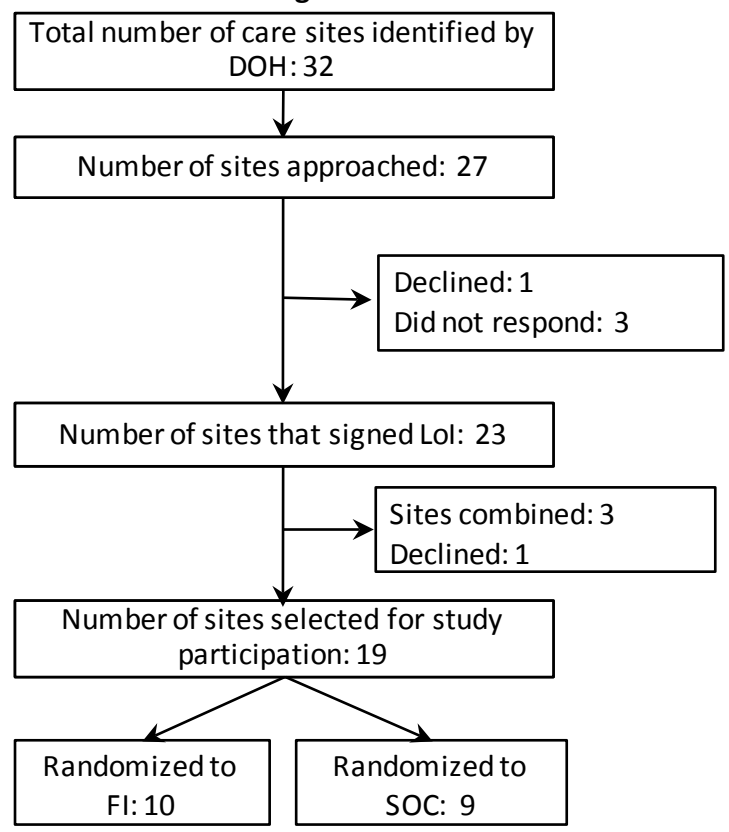

Fig. (1). Selection of test sites. 
Bronx NY
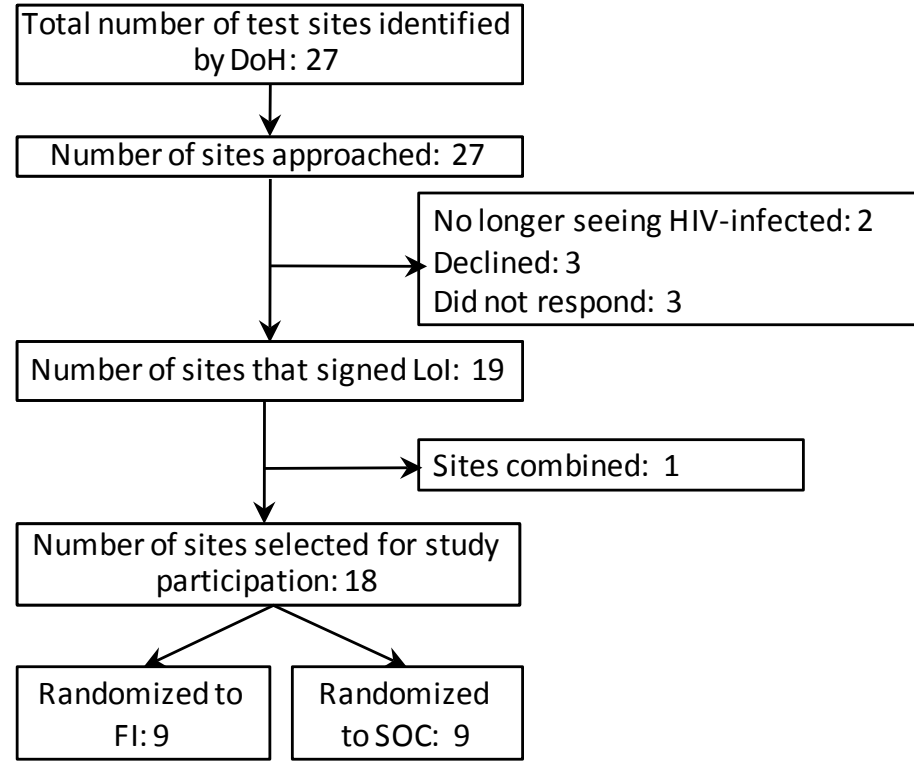

Washington D.C.

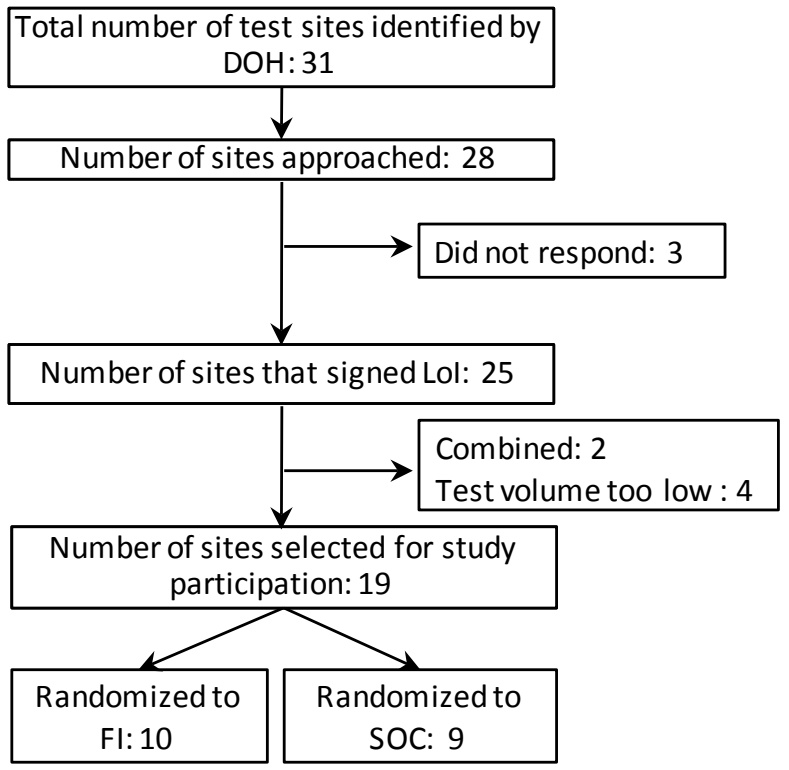

Fig. (2). Selection of care sites.

10 cases. The median proportion of newly identified HIV cases linked to care within three months of initial diagnosis in 2008 in the Bronx sites was 69\%, (interquartile range(IQR) 50\%-86\%); in Washington, D.C., for cases diagnosed in 2009 , the median proportion linked to care was 54\% (IQR 33\%-71\%).

\section{VL Suppression Component}

Table 2 shows the number of HIV-infected patients with care visits (i.e., CD4 or VL measures in the calendar year) and proportion of patients whose last VL was suppressed based on 2008 surveillance data for the Bronx and 2009 surveillance data for Washington, D.C. Five sites in the Bronx and two in Washington, D.C. had in excess of 1000 HIV-infected patients with care visits, 14 and 9 sites respectively had between 100 and 1000 patients, and the remaining sites had fewer than 100 patients. The proportion of patients with VL suppression at their most recent visit was similar across care sites, with median rates of VL suppression in site populations of 57\% (IQR 53\%-61\%) in the Bronx and 64\% (IQR 55\%-72\%) in Washington, D.C.

The 20 participating HIV care sites in the Bronx registered approximately $12,500 \mathrm{HIV}$-infected patients in the 2008 surveillance data; the 19 participating sites in Washington, D.C. registered approximately 5,900 HIVinfected cases in the 2009 surveillance data. In the Bronx, the median number of patients per clinic with a care visit in 2008 at the 20 participating sites was 251, ranging from 83 to 2,751 . In Washington DC, the median number of patients per clinic with a care visit was 153 , ranging from 8 to 1,425 in the 19 clinics.

\section{Study Power}

Study power calculations were based on surveillance data from 2007 for the Bronx and 2008 for Washington, DC (data not shown). Sample size calculation for the site randomized designs used the approach described by Thomson, Hayes and
Cousens [23, 24]. Assuming 20 sites per arm, a mean number of HIV-positive cases of 100 per testing site over the two year period, and an intraclass correlation of 0.31 , the study would have $80 \%$ power to detect an increase from $67 \%$ to $80 \%$ linked to care within 3 months. The intraclass correlation for baseline linkage to care within testing sites in the Bronx was 0.27, within Washington D.C. was 0.31. For care sites, assuming 20 sites per arm, a mean number of cases in care of 180 patients, and an intraclass correlation of 0.11 , the study would have $80 \%$ power to detect an increase in patients with viral suppression from $60 \%$ to $66 \%$ between the standard of care and FI clinics. The intraclass correlation for baseline viral suppression within care sites in the Bronx was 0.07 , within Washington D.C. was 0.10.

\section{DISCUSSION}

The National HIV surveillance system has provided invaluable information for the monitoring of the US epidemic since its inception, including insights on the changing demographics and transmission categories, changes in prevalence and mortality, and estimation of HIV incidence $[12,23,24]$. A study in the San Francisco DOH found that monitoring of VL and CD4 count evaluations from laboratory reporting increased detection of initial primary care visits by $25 \%$ above standard HIV public health investigation [19], indicating that complete reporting of laboratory data to the DOH offers increasingly complete capture for monitoring care visits in known HIV cases. HIV surveillance data have also been used to assess the size of the diagnosed HIV-infected populations currently living in defined jurisdictions, and to map ecologic trends in newly diagnosed infections and transmission potential in a community based on the measured and imputed aggregate mean VL of the HIV-infected population [25, 26]. In addition, increasing confidence in the surveillance data has led the CDC to support local health departments to initiate programs to increase HIV testing and strengthen linkage-tocare and treatment. 
Table 1. Number of Cases and Linkage to Care at Baseline for Participating HIV Testing Sites in Bronx, N.Y. and Washington, D.C.

\begin{tabular}{|c|c|c|c|c|}
\hline \multirow[b]{2}{*}{ Site } & \multicolumn{2}{|c|}{ The Bronx (2008) } & \multicolumn{2}{|c|}{ Washington, D.C. (2009) } \\
\hline & $\begin{array}{l}\text { Number of Newly } \\
\text { Identified HIV-Infected } \\
\text { Individuals in } 1 \text { Year* }\end{array}$ & $\begin{array}{l}\text { Baseline Rates of Linkage-to-Care: } \\
\text { Initiating Care within } 3 \text { Month of } \\
\text { HIV Diagnosis, Data for } 1 \text { Year }\end{array}$ & $\begin{array}{l}\text { Number of Newly } \\
\text { Identified HIV-Infected } \\
\text { Individuals in } 1 \text { Year* }\end{array}$ & $\begin{array}{l}\text { Baseline Rates of Linkage-to-Care: } \\
\text { Initiating Care within } 3 \text { Months of } \\
\text { HIV Diagnosis, Data for } 1 \text { Year }\end{array}$ \\
\hline 1 & 110 & $89 \%$ & 186 & $51 \%$ \\
\hline 2 & 103 & $89 \%$ & 117 & $45 \%$ \\
\hline 3 & 54 & $85 \%$ & 98 & $61 \%$ \\
\hline 4 & 51 & $82 \%$ & 88 & $32 \%$ \\
\hline 5 & 47 & $81 \%$ & 45 & $42 \%$ \\
\hline 6 & 35 & $91 \%$ & 43 & $58 \%$ \\
\hline 7 & 32 & $72 \%$ & 24 & $0 \%$ \\
\hline 8 & 30 & $67 \%$ & 21 & $33 \%$ \\
\hline 9 & 18 & $67 \%$ & 21 & $0 \%$ \\
\hline 10 & 7 & $100 \%$ & 20 & $70 \%$ \\
\hline 11 & 4 & $50 \%$ & 19 & $74 \%$ \\
\hline 12 & 4 & $50 \%$ & 18 & $83 \%$ \\
\hline 13 & 4 & $0 \%$ & 14 & $57 \%$ \\
\hline 14 & 2 & $50 \%$ & 3 & $100 \%$ \\
\hline 15 & 2 & $50 \%$ & 3 & $0 \%$ \\
\hline 16 & 2 & $50 \%$ & 1 & $100 \%$ \\
\hline 17 & 0 & $\mathrm{n} / \mathrm{a}$ & 0 & $\mathrm{n} / \mathrm{a}$ \\
\hline 18 & 0 & $\mathrm{n} / \mathrm{a}$ & 0 & $\mathrm{n} / \mathrm{a}$ \\
\hline 19 & & & 0 & $\mathrm{n} / \mathrm{a}$ \\
\hline Total & 505 & $\begin{array}{c}\text { Median 69\% } \\
(\text { IQR 50\%-86\%) }\end{array}$ & 721 & $\begin{array}{c}\text { Median 54\% } \\
(\text { IQR 33\%-71\%) }\end{array}$ \\
\hline
\end{tabular}

* Sites were initially selected using 2007 data from New York City and 2008 data from Washington D.C. At the time of randomization more recent data was available in New York City (2008) and Washington D.C. (2009), and used in selecting a randomization. Several sites had new cases reported in the selection data but no newly diagnosed infections reported in the more recent randomization data.

Conducting site-randomized trials is a complex undertaking, requiring careful discernment to define the cohort to be assessed and to establish data systems for measuring site-level outcomes [27]. Surveillance data systems can provide comprehensive and accurate estimates of site-level measures and key epidemic indicators for cases that make such trials more operationally feasible and potentially less expensive, especially if utilizing the complete capture of laboratory-based state and local public health surveillance systems.

Surveillance data can only be used to evaluate public health interventions in which study outcomes are aggregated across patients because no individual patient data that originate in surveillance can be used for patient-level research. Measurement of an intervention effect requires outcomes that are reliably captured within the surveillance population (e.g., laboratory measures and events at diagnosis in known cases of HIV infection) and can be aggregated across the chosen site-randomized patient population. Thus, for example, surveillance data cannot measure the impact of an intervention to increase HIV testing at a site, because the number of HIV tests at a site and the HIV-negative results are not captured in the HIV case surveillance system.

A significant advantage of using existing, ongoing data systems is standardized, reproducible baseline data that can be used to inform design parameters of a site-randomized trial. This can significantly increase the power of the study to detect an effect [28]. Baseline levels of study outcomes can be used to conduct a randomization with a higher probability of balance, protecting both power and Type I error. Estimates of the intraclass correlation, a parameter rarely available at the design stage of a site-randomized trial, also help ensure accurate power assessment in the trial. In the HPTN 065 viral suppression component, for example, the relative consistency of baseline proportions of VL suppression suggest that the study is likely to have adequate power to detect an $6 \%$ increase in VL suppression. In comparison, the variability among test sites in both numbers of new cases and linkage-to-care proportions presents a scenario where the study could only reliably detect an increase greater than $13 \%$. However, it must be cautioned that the 1-2 year lag between the baseline surveillance data and trial implementation make the randomization vulnerable 
Table 2. Number of Cases and HIV Plasma Viral Load Suppression ( $<400$ Copies $/ \mathrm{mL})$ at Baseline for Participating HIV Care Sites in Bronx, N.Y. and Washington, D.C.

\begin{tabular}{|c|c|c|c|c|}
\hline Site & \multicolumn{2}{|c|}{ The Bronx, NY(2008) } & \multicolumn{2}{|c|}{ Washington, D.C. (2009) } \\
\hline 2 & $2,500 *$ & $69 \%$ & 1,138 & $58 \%$ \\
\hline 3 & 1,483 & $60 \%$ & 675 & $59 \%$ \\
\hline 5 & 1,118 & $56 \%$ & 376 & $61 \%$ \\
\hline 6 & 702 & $50 \%$ & 321 & $66 \%$ \\
\hline 7 & 379 & $58 \%$ & 319 & $73 \%$ \\
\hline 8 & 376 & $56 \%$ & 296 & $89 \%$ \\
\hline 12 & 210 & $39 \%$ & 98 & $30 \%$ \\
\hline 13 & 180 & $66 \%$ & 67 & $64 \%$ \\
\hline 14 & 173 & $55 \%$ & 60 & $63 \%$ \\
\hline 15 & 131 & $63 \%$ & 41 & $73 \%$ \\
\hline 16 & $130 *$ & $45 \%$ & 33 & $36 \%$ \\
\hline 17 & 119 & $36 \%$ & 15 & $67 \%$ \\
\hline 18 & 118 & $39 \%$ & 12 & $50 \%$ \\
\hline
\end{tabular}

*Site reported patient volume.

${ }^{1}$ VL suppression $(<400$ copies $/ \mathrm{mL})$ achieved at last visit in calendar year specified.

to unmeasured and/or as-yet-unknown administrative and mission changes in HIV test and care sites.

The standardization of surveillance systems across the US permits "ecologic" comparisons of trends in nonintervention cities compared to observed trends in the intervention communities in HPTN 065. With a national surveillance system, the expense of implementing studyspecific data collection in other cities is avoided, although resources are needed for additional programming to implement the study-specific definitions of cohorts, outcomes, and evaluation periods at the non-intervention DOH.

While using surveillance systems has many advantages, to achieve high completeness in study outcome measures may require a significant reporting delay. All data systems experience reporting lag, but the passive nature of surveillance leads to a longer lag than in dedicated trial data collection systems, potentially resulting in a significant delay before the reporting of interim and final trial results. It is typical for annual surveillance data reporting new diagnoses of HIV to be finalized after one year, as reflected in the use of 2008 and 2009 data for study implementation in 2010 and 2011. The most significant lag currently occurs with verification of new diagnoses: the laboratory report that identifies an individual as a possible new diagnosis of HIV must be de-duplicated and matched to the national surveillance registry and, if not matched, a field investigation completed to confirm the date and disposition of diagnosis and collect all other data required for surveillance. Both $\mathrm{CDC}$ and the state and local surveillance authorities have determined that a nine-month period between the date of initial HIV diagnosis (the draw date of the blood for the test that confirmed the HIV diagnosis) to confirmation of diagnosis and entry into the registry is required before reporting is $>90 \%$ complete. However, reporting of laboratory data to update an existing registry record of patient, used for the viral suppression component of HPTN 065 , is considerably more rapid. For example, reporting is complete within three months of draw date for laboratory results (CD4 and/or VL) from known HIV patients in jurisdictions with electronic laboratory reporting. Case migration is also a challenge: there is inevitably a lag in resolving data, particularly date of initial diagnosis, from 
cases relocated from elsewhere, or cases accessing care in the study jurisdiction but residing in a different jurisdiction. De-duplication across jurisdictions of data captured in the state and local surveillance systems occurs through the national CDC database.

A known challenge in assessing aggregate site outcomes is the requirement for specificity and completeness of site location fields on the laboratory requisition that allows surveillance to track laboratory results back to the ordering site. This relies on the procedures of participating sites, and coordination between providers and the DOH. While our success in obtaining and utilizing site-specific data for baseline measures establishes proof of concept for measuring site-level outcomes through the HIV surveillance systems, the data were known to imperfectly identify site for a substantial fraction of laboratory reports; for example, selfreported baseline data were used in the design stage for two care sites that are part of a large multi-site care system because of known problems with identifying the specific site from which the laboratory specimen emanated in the surveillance data. Improvements in the consistency of site identifying fields will be required to ensure accurate attribution of data to specific site locations for HPTN 065 study outcomes, as lack of precision will threaten the ability to detect change resulting from the interventions. Additional staffing resources for acquiring site attribution for study outcomes are being provided to the Departments of Health of participating jurisdictions.

Use of the surveillance data to assess aggregate site outcomes would not be possible in jurisdictions without reporting of all laboratory values, with poor quality monitoring of laboratory data, or with large data entry backlogs. All states have mandatory name-based laboratory reporting of positive HIV test results, but only 28 states, Washington, D.C. and Puerto Rico, currently have mandatory reporting of all CD4 and VL values; some states only require $\mathrm{CD} 4<200$ or detectable VL to be reported. Some jurisdictions, including New York City, also have mandated electronic reporting to the $\mathrm{DOH}$, which is clearly an advantage for achieving complete and timely reporting. Many jurisdictions are improving their systems to utilize electronic reporting from laboratories to ease the upload of data into the surveillance system. HPTN 065 is evaluating the use of surveillance data in an additional four communities that serve as non-intervention communities in this study (Houston, TX Miami, FL; Chicago, IL; Philadelphia, PA). By evaluating the issues encountered across a broad range of cities affected by the HIV epidemic, we plan to assess the human and technical resources required to use surveillance data for evaluating public health strategies in site-randomized studies.

HPTN 065 benefits from extensive CDC investment in the DOHs HIV surveillance systems of each of these six communities: Each jurisdiction has created and executed HIV testing campaigns in the community, taking advantage of additional funding opportunities available through the CDC following the publication of the revised CDC testing recommendations in 2006 [29]. A collateral benefit of these CDC- funded activities is good working relationships between DOH and HIV care and testing providers, and detailed prior knowledge of HIV test and care facilities throughout the community. This is reflected in the high participation rates amongst selected sites in both intervention cities in our study.

\section{CONCLUSION}

Name-based HIV surveillance systems with comprehensive laboratory reporting of HIV-related tests have led to the ability to assess strategies using site level outcomes, offering a new opportunity to conduct program evaluations outside of the specialized research sites that are typically required to measure intervention effects. A siterandomized trial can be designed and implemented that uses a systematic and comprehensive assessment of site-level impact, and assessment can be achieved using data from surveillance systems without incurring the full complexity of establishing an independent data collection system for the study.

HPTN 065 (TLC-Plus) is taking a novel approach to evaluating implementation programs using a site-randomized design and relying on existing surveillance data for assessing outcomes. This approach has great potential for future evaluation of implementation strategies in the U.S. and other countries with similar surveillance systems.

\section{TRIAL REGISTRATION}

ClinicalTrials.gov NCT01152918

\section{ACKNOWLEDGEMENT}

The authors acknowledge the support of the HIV Prevention Trials Network (HPTN) and funding by NIAID, NIDA, and NIMH under Cooperative Agreement \# UM1 AI068619 and \#UM1 AI068617 with the Fred Hutchinson Cancer Research Center. Appreciation is also expressed for the leadership and staff of the participating Departments of Health and the Centers of Disease Control and Prevention. The authors conceived, designed and executed the study, Donnell, Torian, Hall and Griffin were responsible for acquisition of data and the analysis and Donnell, Beauchamp, Torian, Hall, Branson, El Sadr for the interpretation of data.

\section{CONFLICT OF INTEREST}

The authors confirm that this article content has no conflicts of interest.

\section{DISCLAIMER}

The findings and conclusions in this study are those of the authors and do not necessarily represent the views of the Centers for Disease Control and Prevention.

\section{REFERENCES}

[1] Attia S, Egger M, Muller M, Zwahlen M, Low N. Sexual transmission of HIV according to viral load and antiretroviral therapy: systematic review and meta-analysis. AIDS 2009; 23(11): 1397-404.

[2] Donnell D, Baeten JM, Kiarie J, et al. Heterosexual HIV-1 transmission after initiation of antiretroviral therapy: a prospective cohort analysis. Lancet 2010; 375(9731): 2092-8.

[3] Cohen MS, Chen YQ, McCauley M, et al. Prevention of HIV-1 infection with early antiretroviral therapy. N Engl J Med 2011; 365(6): 493-505.

[4] Law MG, Prestage G, Grulich A, Van de Ven P, Kippax S. Modelling the effect of combination antiretroviral treatments on HIV incidence. AIDS 2001; 15(10): 1287-94. 
[5] Blower SM, Gershengorn HB, Grant RM. A tale of two futures: HIV and antiretroviral therapy in San Francisco. Science 2000; 287(5453): 650-4.

[6] Granich RM, Gilks CF, Dye C, De Cock KM, Williams BG. Universal voluntary HIV testing with immediate antiretroviral therapy as a strategy for elimination of HIV transmission: a mathematical model. Lancet 2009; 373(9657): 48-57.

[7] Lima VD, Johnston K, Hogg RS, et al. Expanded access to highly active antiretroviral therapy: a potentially powerful strategy to curb the growth of the HIV epidemic. J Infect Dis 2008; 198(1): 59-67.

[8] Dieffenbach CW, Fauci AS. Universal voluntary testing and treatment for prevention of HIV transmission. JAMA 2009; 301(22): 2380-2.

[9] Marks G, Crepaz N, Senterfitt JW, Janssen RS. Meta-analysis of highrisk sexual behavior in persons aware and unaware they are infected with HIV in the United States: implications for HIV prevention programs. J Acquir Immune Defic Syndr 2005; 39(4): 446-53.

[10] Graham SM, Holte SE, Peshu NM, et al. Initiation of antiretroviral therapy leads to a rapid decline in cervical and vaginal HIV-1 shedding. AIDS 2007; 21(4): 501-7.

[11] Vergidis PI, Falagas ME, Hamer DH. Meta-analytical studies on the epidemiology, prevention, and treatment of human immunodeficiency virus infection. Infect Dis Clin North Am 2009; 23(2): 295-308.

[12] Campsmith ML, Rhodes PH, Hall HI, Green TA. Undiagnosed HIV prevalence among adults and adolescents in the United States at the end of 2006. J Acquir Immune Defic Syndr 2010; 53(5): 619-24.

[13] Vital signs: HIV testing and diagnosis among adults--United States, 2001-2009. MMWR Morb Mortal Wkly Rep 2010; 59(47): 1550-5.

[14] HIV prevalence estimates--United States, 2006. MMWR Morb Mortal Wkly Rep 2008; 57(39): 1073-6.

[15] Torian LV, Wiewel EW, Liu KL, Sackoff JE, Frieden TR. Risk factors for delayed initiation of medical care after diagnosis of human immunodeficiency virus. Arch Intern Med 2008; 168(11): 1181-7.

[16] Giordano TP, Gifford AL, White AC Jr, et al. Retention in care: a challenge to survival with HIV infection. Clin Infect Dis 2007; 44(11): 1493-9.
[17] Torian LV, Wiewel EW. Continuity of HIV-related medical care, New York City, 2005-2009: Do patients who initiate care stay in care? AIDS Patient Care STDS 2011; 25(2): 79-88.

[18] Marks G, Gardner LI, Craw J, Crepaz N. Entry and retention in medical care among HIV-diagnosed persons: a meta-analysis. AIDS 2010; 24(17): 2665-78.

[19] Zetola NM, Bernstein K, Ahrens K, et al. Using surveillance data to monitor entry into care of newly diagnosed HIV-infected persons: San Francisco, 2006-2007. BMC Public Health 2009; 9: 17

[20] Meyerson BE, Klinkenberg WD, Perkins DR, Laffoon BT. Who's in and who's out: use of primary medical care among people living with HIV. Am J Public Health 2007; 97(4): 744-9.

[21] Torian LV, Henning KJ, Kellerman SE, Frieden TR. Striving toward comprehensive HIV/AIDS surveillance: the view from New York City. Public Health Rep 2007; 122(Suppl 1): 4-6.

[22] Hayes RJ, Moulton L. Cluster Randomised Trials. UK: Chapman \& Hall/CRC Interdisciplinary Statistics 2009.

[23] Hayes RJ, Bennett S. Simple sample size calculation for clusterrandomized trials. Int J Epidemiol 1999; 28(2): 319-26.

[24] Thomson A, Hayes R, Cousens S. Measures of between-cluster variability in cluster randomized trials with binary outcomes. Stat Med 2009; 28(12): 1739-51.

[25] Das M, Chu PL, Santos GM, et al. Decreases in community viral load are accompanied by reductions in new HIV infections in San Francisco. PLoS One 2010; 5(6): e11068.

[26] Des Jarlais DC, Arasteh K, Perlis T, et al. Convergence of HIV seroprevalence among injecting and non-injecting drug users in New York City. AIDS 2007; 21(2): 231-5.

[27] Donnell D, Hughes JP, Fleming TR. Challenges in the design of HIV prevention trials in the United States. J Acquir Immune Defic Syndr 2010; 55(S2): S136-S40.

[28] Hughes JP. Using baseline data to design a group randomized trial. Stat Med 2005; 24(13): 1983-94

[29] Branson BM, Handsfield HH, Lampe MA, et al. Revised recommendations for HIV testing of adults, adolescents, and pregnant women in health-care settings. MMWR Recomm Rep 2006; 55(RR14): $1-17$.

(C) Donnell et al.; Licensee Bentham Open.

This is an open access article licensed under the terms of the Creative Commons Attribution Non-Commercial License (http://creativecommons.org/licenses/by-nc/ $3.0 /$ ) which permits unrestricted, non-commercial use, distribution and reproduction in any medium, provided the work is properly cited. 